\title{
ỨNG DỤNG KỸ THUÂTT CHỤP CẮT LỚP 2 MỨC NĂNGG LƯƠNG ĐỂ PHÂN TÍCH, DỰ BÁO TÍNH CHẤT CƠ LÝ CỦA MẪU LÕI
}

\author{
Nguyễn Lâm Quốc Cường, Nguyễn Hồng Minh \\ Viện Dầu khí Việt Nam \\ Email: cuongnlq@vpi.pvn.vn \\ https://doi.org/10.47800/PVJ.2021.02-03
}

\section{Tóm tắt}

Hơn 20 năm qua, công nghệ chụp cắt lớp được sử dụng trong ngành công nghiệp dầu khí đểnghiên cứu đặc tính mẫu lõi và trực quan hóa dòng chảy lưu chất [1 - 8]. Trong đó, chụp cắt lớp định tính cung cấp thông tin về tính bất đồng nhất [9], sự thay đổi thạch học, khe nứt, hang hốc và mức độ xâm nhập của dung dịch khoan. Chụp cắt lớp định lượng ngoài các ứng dụng trên còn dùng để tính mật độ tổng (bulk density), độ rỗng và hiệu chuẩn đường cong địa vật lý giếng khoan.

Bài báo giới thiệu phương pháp dự báo tính chất đá vỉa (như mật độ hạt, độ rỗng. ...) từ kết quả chụp cắt lớp theo phương pháp chụp cắt lớp 2 mức năng lượng (Dual-Energy CT Scanning - DECT) tại 140 kV và 80 kV. Kết quả chụp cắt lớp được so sánh với giá trị đường cong địa vật lý, cho thấy tiềm năng lớn trong việc xác định các tính chất vật lý của đá chứa cũng như chất lưu vỉa.

Từ khóa: Chụp cắt lớp 2 mức năng lượng, đặc tính đá chứa, tán xạ Compton, hiệu ứng quang điện, độ rỗng.

\section{Giới thiệu}

Chụp cắt lớp là kỹ thuật xử lý hình ảnh không phá hủy mẫu, tận dụng công nghệ tia $X$ và các thuật toán tái tạo để xây dựng các mặt cắt ngang và dọc của vật thể. Không chỉ ứng dụng trong y tế, chụp cắt lớp đã được sử dụng để nghiên cứu đất đá vỉa trong ngành công nghiệp dầu khí hơn 20 năm qua [1 - 8]. Về cơ bản, chụp cắt lớp trong ngành công nghiệp dầu khí có thể chia làm 2 hướng chính là mô tả đặc tính mẫu lõi và trực quan hóa dòng chảy lưu chất.

Ứng dụng kỹ thuật chụp cắt lớp trong mô tả đặc tính mẫu lõi gồm việc chụp các mẫu đường kính lớn và mẫu lõi đường kính nhỏ (plug). Chụp cắt lớp trong điều kiện mẫu bảo quản rất hữu ích cho các mẫu không cố kết hay những mẫu thí nghiệm mà không làm ảnh hưởng đến trạng thái dính ướt của mẫu. Chụp cắt lớp định tính cung cấp thông tin về tính bất đồng nhất [9], sự thay đổi thạch học, khe nứt, hang hốc và mức độ xâm nhập của dung dịch khoan. Chụp cắt lớp định lượng ngoài các ứng dụng trên còn dùng để tính mật độ tổng, độ rỗng và hiệu chuẩn đường cong địa vật lý giếng khoan.

Ngày nhận bài: 27/10/2020. Ngày phản biện đánh giá và sửa chữa: 27/10 - 16/11/2020. Ngày bài báo được duyệt đăng:2/2/2021.
Phương pháp chụp cắt lớp được dùng nhiều trong việc trực quan hóa dòng chảy chất lưu bằng cách sử dụng chất đánh dấu phóng xạ (dopants) để theo dõi sự dịch chuyển lưu chất bên trong mẫu lōi thông qua quá trình bơm ép. Chất đánh dấu này tạo ra độ tương phản giữa các pha lưu chất giúp quan sát và định lượng sự thay đổi cũng như phân bố độ bão hòa chất lưu trong mẫu, quan sát hiệu ứng trọng lực và hiệu ứng ngón tay, hiện tượng chất lưu bị bẫy lại hay trượt qua, hiệu ứng bất đồng nhất của dòng chảy. Chụp cắt lớp là công cụ rất tốt để quan sát hiệu quả việc xử lý lưu chất như: acid, gel, polymer, hơi nóng và bọt cùng với việc giữ mẫu trong điều kiện vỉa bằng thiết bị giữ mẫu chuyên dụng. Khi tiến hành thí nghiệm dòng chảy 3 pha, chụp cắt lớp cung cấp giải pháp hiệu quả để tính toán các độ bão hòa tại chỗ riêng lẻ $[1,10,11]$.

Năm 1987, Wellington và Vinegar [1] lần đầu tiên chỉ ra tương quan của hình ảnh chụp cắt lớp với mật độ tổng (bulk density) và số nguyên tử (atomic number) dựa trên quan sát việc quét mẫu ở mức năng lượng cao (trên 100 kV) - khi hiệu ứng tán xạ Compton chiếm ưu thế và năng lượng thấp (dưới 100 kV) - khi hiệu ứng hấp thụ quang điện chiếm ưu thế. Kỹ thuật chụp cắt lớp 2 mức năng lượng được áp dụng cho mẫu lõi có đường kính nhỏ (plug) và đường kính lớn (full diameter) với 
khoảng cách giữa các lát cắt cần xử lý là $5 \mathrm{~mm}$ để giảm khối lượng số liệu cần xử lý. Bài báo này tập trung vào dự báo tính chất mẫu lôi bằng cách sử dụng kỹ thuật chụp cắt lớp 2 mức năng lượng.

\section{Chụp cắt lớp 2 mức năng lượng (DECT)}

Khi chụp cắt lớp 2 mức năng lượng, mẫu sẽ được quét 2 lần cùng vị trí nhưng sử dụng 1 mức năng lượng cao và 1 mức năng lượng thấp. Cách thiết lập mức năng lượng cao và thấp có thể tận dụng lợi thế tương tác của 2 hiện tượng chính của tia $X$ là sự hấp thụ quang điện (chiếm ưu thế khi dùng mức năng lượng thấp) và hiệu ứng tán xạ Compton (chiếm ưu thế khi dùng mức năng lượng cao), tương ứng với sự phụ thuộc vào số nguyên tử và mật độ electron. Xác suất tán xạ Compton phụ thuộc vào năng lượng tia $X$ và mật độ electron, trong khi đó xác suất hấp thụ quang điện tăng nhanh theo số nguyên tử và giảm nhanh khi tăng năng lượng photon. Vi vậy, từ việc đo mức độ suy giảm chùm tia $X$ của 2 mức năng lượng khác nhau có thể giúp tìm ra lượng tán xạ Compton và lượng hấp thụ quang điện khi truyền qua vật liệu [12]. Từ đó có thể tính số nguyên tử hiệu dụng $\left(Z_{\text {eff }}\right)$ và mật độ electron của đối tượng mẫu bằng cách quét 2 mức năng lượng tách biệt.

Chụp cắt lớp 2 mức năng lượng được sử dụng rộng rãi trong y tế để đo mật độ khoáng chất của xương sau khi loại bỏ hiệu ứng hấp thụ tia $X$ của các mô mềm. Kỹ thuật 2 mức năng lượng cũng được ứng dụng trong quét hành lý để kiểm tra các vật liệu không chính thống bằng biểu đồ giữa $Z_{\text {eff }}$ và mật độ [13].

Wellington và Vinegar [1] đề nghị dùng 100 kV như là giá trị đầu vào cho 2 hiệu ứng của máy chụp cắt lớp (trên 100 kV dành cho tán xạ Compton, dưới 100 kV dành cho hấp thụ quang điện). Một số máy chụp cắt lớp ngày nay có mức phát tối đa là $140 \mathrm{kV}$ nên dùng mức năng lượng cao để quét. Còn lại có thể dùng các mức dưới 100 kV như $90,80,70$ hay $60 \mathrm{kV}$ để làm mức năng lượng thấp nhưng thông thường thì dùng $80 \mathrm{kV}$.

\subsection{Công thức dùng trong chụp cắt lớp 2 mức năng lượng}

Phương trình được trích dẫn rộng rãi dùng cho $\mathrm{DECT}$ được công bố bởi Wellington và Vinegar [1].

$$
\mu=\rho\left[a+\frac{b Z^{n}}{E^{3,2}}\right]
$$

Trong đó:

$\mu$ : Hệ số suy giảm tuyến tính;

$\rho:$ Mật độ electron;
E: Năng lượng tia $X$;

Z: Số nguyên tử;

a: Hệ số Klein-Nishina,

b: Hằng số;

n: Số mũ của Z.

Mật độ electron $\rho$ liên quan đến mật độ tổng $\rho_{b}$ theo công thức sau dành cho các loại vật liệu [14].

$$
\rho_{b}=1,0704 \rho-0,1883
$$

Số mũ $\mathrm{n}$ cũng được dùng để tính số nguyên tử hiệu dụng của tổ hợp như công thức (3) mà trong đó $Z_{\mathrm{i}}$ là số nguyên tử của nguyên tố thứ $\mathrm{i}$ trong tổ hợp và $\mathrm{f}_{\mathrm{i}}$ là tỷ lệ của nguyên tử đại diện cho nguyên tố thứ i trong tổ hợp.

$$
Z_{\text {eff }}=\left[\sum f_{i} Z_{i}^{n}\right]^{1 / n}
$$

Mặc dù nghiên cứu của Wellington và Vinegar sử dụng giá trị $\mathrm{n}=3,8$, nhứng có một số nghiên cứu khác dùng giá trị 3,6 , có khi là 3 . Bài báo này dùng giá trị $n=3,8$ cho $n$ như đa số và cũng phù hợp với số mũ dùng trong phân tích đường cong [14 - 17]. Bảng 1 thể hiện một vài giá trị $Z_{\text {eff }}$ và giá trị mật độ tổng tương ứng của một số vật liệu phổ biến cần cho tính toán khi quét 2 mức năng lượng [17].

$Z_{\text {eff }}$ liên quan đến hệ số quang điện, $P_{\mathrm{e}}$ như công thức (4).

$$
P_{e}=\left[\frac{Z_{e f f}}{10}\right]^{3,8}
$$

Giống như $\mathrm{P}_{\mathrm{e}^{\prime}}$ giá trị $\mathrm{Z}_{\text {eff }}$ cũng ít thay đổi theo độ rỗng. Các nghiên cứu cho thấy $Z_{\text {eff }}$ của calcite, dolomite và quartz chỉ thay đổi $5 \%$ khi độ rỗng thay đổi $35 \%$ với nước là chất lưu trong lỗ rỗng. Vi vậy, $Z_{\text {eff }}$ cho thấy tiềm năng là chỉ số thạch học rất tốt và thông qua mối quan hệ được

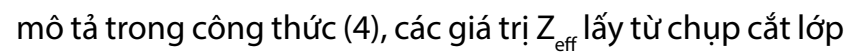
có thể được chuyển đổi thành giá trị $\mathrm{P}_{\mathrm{e}}$ để ước tính sơ bộ sự xuất hiện của khoáng vật trong mẫu lỗi.

Do tính chất phi tuyến phức tạp của công thức (1) nên khó để tính $Z_{\text {eff }}$ và $\rho$ tại các vị trí lát cắt. Angulo và Ortiz [18] đã sử dụng mô hình đa thức để định lượng 2 giá trị này từ ảnh CT, mặc dù kết quả bị ảnh hưởng bởi kỹ thuật tái tạo CT như hiệu ứng cứng chùm tia gây ra bởi tính đa sắc của tia X. Vi vậy, trong các đối tượng mẫu nhiều thành phần, Wellington và Vinegar đã đề cập đến sự cần thiết của việc loại bỏ hiện tượng cứng chùm tia trong quá trình tái cấu trúc 2 mức năng lượng để đạt được kết quả tốt bằng cách áp dụng lọc tia và xử lý trước khi tái tạo. 
Bảng 1. Giá trị mật độ tổng và sốnguyên tử hiệu dụng của một số khoáng vật

\begin{tabular}{|c|c|c|c|}
\hline Tên & Công thức hóa học & Mật độ tổng (g/cc) & $Z_{\text {eff }}$ \\
\hline Nước & $\mathrm{H}_{2} \mathrm{O}$ & 1 & 7,5195 \\
\hline Graphite & C & 2,3 & 6 \\
\hline Calcite & $\mathrm{CaCO}_{3}$ & 2,71 & 15,71 \\
\hline Dolomite & $\mathrm{CaMg}\left(\mathrm{CO}_{3}\right)_{2}$ & 2,87 & 13,7438 \\
\hline Air & Air & 0,001 & 7,224 \\
\hline Quartz Mineral & $\mathrm{SiO}_{2}$ & 2,65 & 11,7842 \\
\hline Aluminum Pure & $\mathrm{Al}$ & 2,7 & 13 \\
\hline Iron Pure & $\mathrm{Fe}$ & 5,6 & 26 \\
\hline Anhydrite & $\mathrm{CaSO}_{4}$ & 2,95 & 15,6847 \\
\hline Fused Quartz & $\mathrm{SiO}_{2}$ & 2,2 & 11,7842 \\
\hline Pyrite & $\mathrm{FeS}_{2}$ & 5,02 & 21,9588 \\
\hline Siderite & $\mathrm{FeCO}_{3}$ & 3,96 & 21,0932 \\
\hline Barite & $\mathrm{BaSO}_{4}$ & 4,5 & 47,2008 \\
\hline Na-Feldspar & $\mathrm{NaAlSi}_{3} \mathrm{O}_{8}$ & 2,61 & 11,5534 \\
\hline K-Feldspar & $\mathrm{KAISi}_{3} \mathrm{O}_{8}$ & 2,53 & 13,3895 \\
\hline Kaolinite & $\mathrm{Al}_{2} \mathrm{Si}_{2} \mathrm{O}_{5}(\mathrm{OH})_{4}$ & 2,6 & 11,1622 \\
\hline Illite & $\mathrm{KAl}_{3} \mathrm{Si}_{3} \mathrm{O}_{10}(\mathrm{OH})_{2}$ & 2,8 & 9,6058 \\
\hline Na-Montmorillonite & $\mathrm{NaAl}_{5} \mathrm{MgSi}_{12} \mathrm{O}_{30}(\mathrm{OH})_{6}$ & 2,65 & 11,462 \\
\hline Ca-Montmorillonite & $\mathrm{Ca}_{0,5} \mathrm{Al}_{5} \mathrm{MgSi}_{12} \mathrm{O}_{30}(\mathrm{OH})_{6}$ & 2,65 & 11,8277 \\
\hline Chlorite & $\mathrm{Fe}_{2} \mathrm{Mg}_{2} \mathrm{Al}_{2} \mathrm{Si}_{2} \mathrm{~A}_{12} \mathrm{O}_{10}(\mathrm{OH})_{8}$ & 2,9 & 11,6449 \\
\hline Celestite & $\mathrm{SrSO}_{4}$ & 3,9 & 30,4686 \\
\hline Talc & $\mathrm{Mg}_{3} \mathrm{Si}_{4} \mathrm{O}_{10}(\mathrm{OH})_{2}$ & 2,75 & 8,4538 \\
\hline Rutile & $\mathrm{TiO}_{2}$ & 4,2 & 19,0006 \\
\hline Halite & $\mathrm{NaCl}$ & 2,35 & 15,3295 \\
\hline
\end{tabular}

Các máy chụp cắt lớp chủ yếu cung cấp dữ liệu suy giảm dưới dạng số $\mathrm{CT}$ (CT number, đơn vị là $\mathrm{HU}$ ) được hiệu chỉnh bằng sự suy giảm của nước và được thể hiện như sau:

$$
C T N=\frac{\mu-\mu_{w}}{\mu_{w}} \times 1000
$$

Vinegar và Kehl [14 ] đã rút ra 2 phương trình khác từ phương trình 1 mà sử dụng 2 mức năng lượng thấp và cao như sau:

$$
\begin{gathered}
\rho=A \times C T N_{\text {low }}-B \times C T N_{\text {high }}+C \\
Z_{\text {eff }}=D \times\left[\frac{E \times C T N_{\text {low }}-C T N_{\text {high }}-F}{\rho}\right]^{1 / 3,8}
\end{gathered}
$$

Vinegar và Kehl đề xuất quét qua một số mẫu lõi biết trước mật độ tổng và $Z_{\text {eff }}$ sử dụng kết quả liên kết số $\mathrm{CT}$ (tại 2 mức năng lượng thấp và cao) theo phương trình (6) và (7) để tính ra các giá trị $A, B, C, D, E$ và $F$. Những giá trị này sẽ được áp dụng để tính $\rho_{\mathrm{b}}$ và $Z_{\text {eff }}$ cho những mẫu khác. Một số phần mềm hiện nay có quy trình xử lý tương tự phương pháp của Vinegar và Kehl để tính $\rho$ và $Z_{\text {eff }}$ liên tục trên các lát cắt cho từng điểm ảnh (voxel by voxel).

\section{2. Đơn giản hóa phương trình 2 mức năng lượng}

Quy trình dưới đây được sử dụng để xác định định lượng mật độ tổng $\rho_{\mathrm{b}}$ và $Z_{\text {eff' }}$ dựa trên giá trị số $\mathrm{CT}$ của vùng quan tâm (Region of Interest - ROI) trong khoảng mẫu lõi được đo. Tại vị trí mỗi lát cắt, mức năng lượng quét là $80 \mathrm{kV}$ và $140 \mathrm{kV}$.

Phương trình từ (2) - (6) có thể viết lại như sau:

$$
\rho_{b}=1,0704 \times\left[A \times C T N_{\text {low }}-B \times C T N_{\text {high }}+C\right]-0,1883
$$

Phương trình (8) được sắp xếp lại cho đơn giản hơn chỉ với 3 hệ số mới là $m, p$ và $q$.

$$
\rho_{b}=m \times C T N_{\text {low }}+p \times C T N_{\text {high }}+q
$$

Phương trình (7) được viết lại như sau:

$$
D \times E \times C T N_{\text {low }}-D \times C T N_{\text {high }}-D \times F=\rho \times Z_{\text {eff }}^{3,8}
$$

Phương trình (2) được viết lại như sau:

$$
\rho=0,9342 \times \rho_{b}+0,1759
$$

Kết hợp (2) phương trình (10) và (11) thành phương trình đơn giản hơn với 3 hệ số $r$, s và t như sau:

$r \times \operatorname{CTN}_{\text {low }}+s \times C T N_{\text {high }}+t=\left[0,9342 \times \rho_{b}+0,1759\right] \times Z_{\text {eff }}^{3,8}(12)$

Hoặc

$$
Z_{\text {eff }}=\left[\frac{r \times C T N_{\text {low }}+s \times C T N_{\text {high }}+t}{0,9342 \times \rho_{b}+0,1759}\right]^{1 / 3,8}
$$


Giải các phương trình 3 ẩn này sẽ tìm ra được các giá trị $m, p, q$ và $r, s, t$. Ngoài ra, hệ số quang điện cũng có thể được tính cho mỗi lát cắt dựa trên công thức (4). Điều quan trọng là dữ liệu năng lượng cao và thấp và số $\mathrm{CT}$ phải được thu thập tại cùng một vị trí và vùng quan tâm.

\subsection{Thí nghiệm chụp cắt lớp xác định mật độ tổng}

\subsubsection{Kết quả quét mẫu lõi hình trụ}

Trước tiên, căn chỉnh máy chụp cắt lớp được thực hiện với 3 mẫu chuẩn đã biết trước các thông số $\rho_{\mathrm{b}}$ và $Z_{\text {eff }}$ (có

Bảng 2. Giá trị hệ số cho các mẫu chuẩn

\begin{tabular}{|c|c|}
\hline Hệ số & Giá trị \\
\hline m & $-0,8207$ \\
\hline$p$ & 2,0109 \\
\hline $\mathrm{q}$ & $1.245,9$ \\
\hline $\mathrm{r}$ & 34.683 \\
\hline $\mathrm{s}$ & -44.491 \\
\hline $\mathrm{t}$ & -6.651 .160 \\
\hline
\end{tabular}

thể lựa chọn từ các mẫu tiêu chuẩn ở Bảng 1). Các mẫu này được dùng để tính ra 6 hệ số $m, p, q, r, s$ và t như miêu tả ở trên . Từ giá trị số $C T$ tại 2 mức năng lượng cao và thấp cùng giá trị tương ứng $\rho_{\mathrm{b}}$ và $Z_{\text {eff }}$ của các mẫu hiệu chuẩn đã biết sẽ tính được các hệ số quét 2 mức năng lượng được thể hiện trong Bảng 2.

Mẫu dùng để thí nghiệm được chọn từ các mẫu đại diện cho các loại thạch học khác nhau gồm: đá vôi, đá cát kết và sét phiến. Ba mẫu này được chụp cắt lớp tại mức năng lượng 140 kV và 80 kV tại cùng vị trí. Giá trị số $C T$ tại vùng quan tâm của 2 mức cao và thấp được tính toán sang $\rho_{b}$ và $Z_{\text {eff }}$ theo phương trình (9) và (13) và cho kết quả trong Bảng 3.

Bảng 3 cũng cung cấp giá trị trung bình của $\rho_{\mathrm{b}}$ và $Z_{\text {eff }}$ cho 3 mẫu. Giá trị đo mật độ tổng bằng phương pháp phân tích thông thường có thể tích tổng lần lượt là 1906,2; 2120; 2529,2 mg/cc với sai số hiệu dụng tương ứng là

Bảng 3. Giá trị $\rho_{b}$ và $Z_{\text {eff }}$ khi quét các mẫu nhỏ

\begin{tabular}{|c|c|c|c|c|c|c|}
\hline TT & CTN low & CTN $N_{\text {high }}$ & $\begin{array}{c}\rho_{b} \\
\left(\mathbf{m g} / \mathrm{cm}^{3}\right)\end{array}$ & $Z_{\text {eff }}$ & \multicolumn{2}{|c|}{ Plug } \\
\hline 1 & 3411,5 & 1789,4 & 2044,4 & 14,91 & \multicolumn{2}{|c|}{ Đá vôi } \\
\hline 2 & 3407,5 & 1786,4 & 2041,7 & 14,92 & Avg. $\rho_{b}$ & \\
\hline 3 & 3404,0 & 1784,3 & 2040,4 & 14,92 & 2041,50 & $\mathrm{mg} / \mathrm{cc}$ \\
\hline 4 & 3404,3 & 1785,1 & 2041,6 & 14,91 & Avg. $Z_{\text {eff }}$ & \\
\hline 5 & 3402,2 & 1783,7 & 2040,6 & 14,91 & 14,91 & \\
\hline 6 & 3399,9 & 1782,6 & 2040,3 & 14,91 & & \\
\hline 7 & 3394,4 & 1779,8 & 2039,3 & 14,90 & & \\
\hline 8 & 3393,6 & 1779,9 & 2040,0 & 14,90 & & \\
\hline 9 & 3394,6 & 1782,9 & 2045,2 & 14,87 & & \\
\hline 1 & 2521,5 & 1480,4 & 2153,4 & 11,89 & \multicolumn{2}{|c|}{ Đá cát kết } \\
\hline 2 & 2491,5 & 1458,8 & 2134,6 & 11,90 & Avg. $\rho_{b}$ & \\
\hline 3 & 2527,2 & 1479,4 & 2146,7 & 11,95 & 2147,70 & $\mathrm{mg} / \mathrm{cc}$ \\
\hline 4 & 2526,2 & 1478,0 & 2144,7 & 11,96 & Avg. $Z_{\text {eff }}$ & \\
\hline 5 & 2459,9 & 1437,0 & 2116,8 & 11,90 & 11,93 & \\
\hline 6 & 2552,7 & 1494,7 & 2156,6 & 11,98 & & \\
\hline 7 & 2583,0 & 1516,7 & 2176,0 & 11,97 & & \\
\hline 8 & 2552,4 & 1499,0 & 2165,5 & 11,93 & & \\
\hline 9 & 2503,9 & 1467,3 & 2141,6 & 11,90 & & \\
\hline 10 & 2553,0 & 1498,7 & 2164,4 & 11,93 & & \\
\hline 11 & 2525,8 & 1480,8 & 2150,8 & 11,92 & & \\
\hline 12 & 2538,7 & 1488,4 & 2155,3 & 11,94 & & \\
\hline 13 & 2474,5 & 1448,9 & 2128,6 & 11,88 & & \\
\hline 14 & 2496,1 & 1459,7 & 2132,7 & 11,93 & & \\
\hline & & & & & & \\
\hline 1 & 3377,2 & 2015,0 & 2526,1 & 12,48 & \multicolumn{2}{|c|}{ Đá phiến sét } \\
\hline 2 & 3574,8 & 2139,7 & 2614,8 & 12,57 & Avg. $\rho_{b}$ & \\
\hline 3 & 3546,0 & 2127,3 & 2613,5 & 12,50 & 2610,20 & $\mathrm{mg} / \mathrm{cc}$ \\
\hline 4 & 3512,9 & 2115,0 & 2615,9 & 12,40 & Avg. $Z_{\text {eff }}$ & \\
\hline 5 & 3461,7 & 2091,1 & 2609,9 & 12,29 & 12,45 & \\
\hline 6 & 3580,9 & 2154,5 & 2639,6 & 12,46 & & \\
\hline 7 & 3591,9 & 2165,1 & 2651,9 & 12,43 & & \\
\hline
\end{tabular}



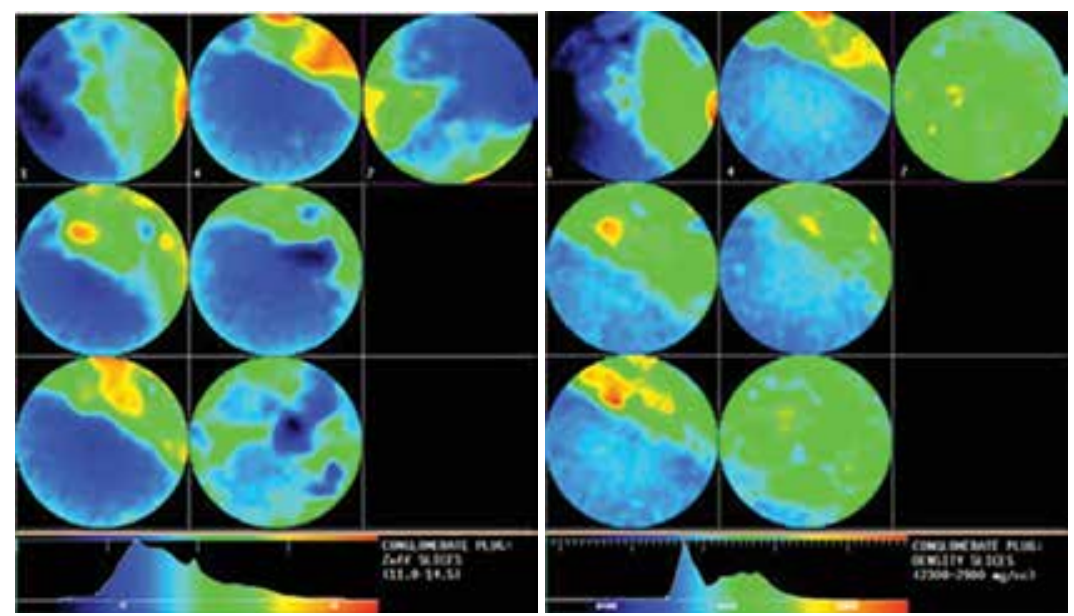

Hinh 1. Giá trị $\rho_{b}$ và $Z_{\text {eff }}$ ủa từng lát căt
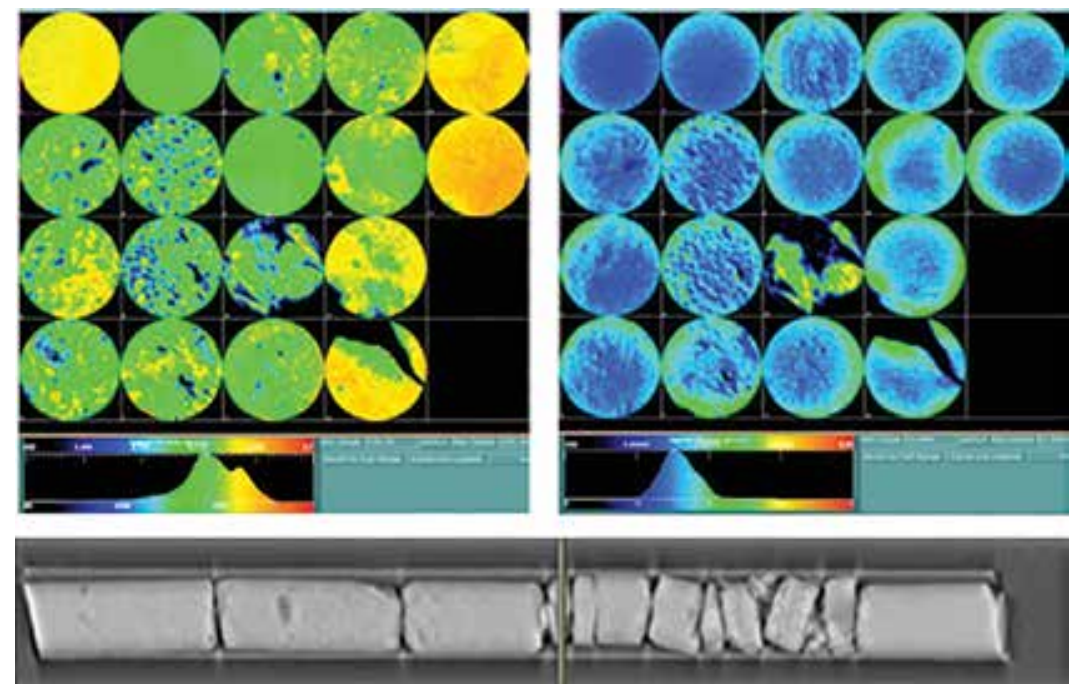

Hình 2. Sự xuất hiện của hiệu ứng cứng chùm tia trên các lớp cắt

$-7,1 \% ;-1,7 \% ;-3,2 \%$ (với kỹ thuật chụp 3 mức năng lượng thì giá trị dự báo cao hơn một chút).

Các giá trị trung bình $\rho_{\mathrm{b}}$ và $\mathrm{Z}_{\text {eff }}$ cho mỗi lát cắt có thể tính toán bằng phần mềm xử lý. Ngoài ra, kỹ thuật đổ màu lên hình ảnh cũng được sử dụng để trực quan hóa số liệu. Hình 1 là hình ảnh được đổ màu - màu sáng đại diện cho các giá trị cao, màu đen đại diện cho các giá trị thấp.

Hình ảnh của các lát cắt rất hữu ích trong việc cung cấp thông tin về tính đồng nhất cũng như cho thấy sự xuất hiện của hiệu ứng cứng chùm tia. Từ đó, có thể loại bỏ các giá trị nhiễu ảnh hưởng đến kết quả (Hình 2).

\subsubsection{Kết quả quét mẫu lõi đường kính lớn}

Thí nghiệm chụp cắt lớp 2 mức năng lượng cũng được áp dụng cho mẫu đường kính lớn. Mẫu được khoan bằng dung dịch gốc nước, bảo quản trong ống nhôm bịt kín 2 đầu để mẫu không bị xê dịch, nứt nẻ do vận chuyển cũng như giữ nguyên được hiện trạng của mẫu. Mẫu được quét tại 2 mức năng lượng 140 kV và 80 kV.

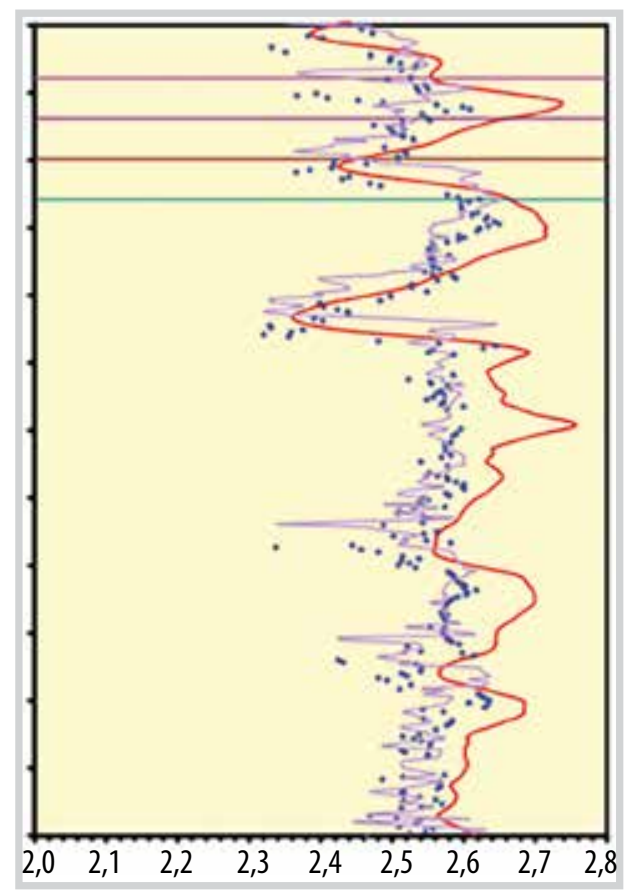

Hình 3. Tương quan của đường cong mật độ (màu đỏ), giá trị chụp cắt lớp rời rạc (chấm tròn) và trung bình hóa (đường tím)

\subsection{So sánh kết quả quét 2 mức năng lượng với dữ liệu đường cong địa vật lý}

Kết quả định lượng từ việc quét 2 mức năng lượng được so sánh với các đường cong địa vật lý giếng khoan. Hình 3 thể hiện sự tương quan của mật độ tổng lấy từ đường cong địa vật lý và từ chụp cắt lớp 2 mức năng lượng (điểm rời rạc và trung bình hóa). Có thể áp dụng để dịch chuyển độ sâu cho phù hợp. So sánh kết quả thu được từ chụp cắt lớp với đường cong địa vật lý cho thấy, do mức năng lượng của việc đo địa vật lý khá cao (từ 200 - 2.000 kV), nên đường cong địa vật lý sẽ chịu ảnh hưởng của hiệu ứng tán xạ Compton là chủ yếu mà không bị ảnh hưởng của hiệu ứng quang điện. Do vậy, dẫn đến việc khó xác định một số khoáng vật như dolomite trong đá carbonate.

\subsection{Thí nghiệm đo độ rỗng dùng kỹ thuật chụp cắt lớp 2 mức năng lượng}

Với kỹ thuật chụp cắt lớp 2 mức năng lượng, độ rỗng của mẫu được tính toán từ giá trị mật độ tổng và số nguyên tử hiệu dụng theo các bước sau: 
- Quét mẫu vật tại cùng vị trí qua 2 mức năng lượng khác nhau;

- Quét mẫu hiệu chuẩn để tạo ra các hệ số chuẩn;

- Tính mật độ tổng và số nguyên tử hiệu dụng cho từng lát cắt dựa trên số $\mathrm{CT}$ của mức năng lượng cao và số CT của mức năng lượng thấp;

- Tính hệ số quang điện $\mathrm{P}_{\mathrm{e}}$ từ $\mathrm{Z}_{\text {eff' }}$;

- Tính mật độ electron từ mật độ tổng;

- Tính U, chỉ số thể tích quang điện hấp thụ dựa trên công thức

$$
U=P_{e} \times \rho
$$

Dùng các biểu đồ tương quan giữa độ rỗng, thạch học và độ bão hòa tiêu chuẩn của Schlumberger để tính tỷ lệ khoáng vật $[16,18,20]$;

Khi tỷ lệ khoáng vật được biết, mật độ khung đá $\rho_{\text {ma }}$ sẽ được tính dựa trên quy tắc trộn lẫn và độ rỗng sẽ được tính theo công thức sau:

$$
\emptyset=\frac{\rho_{m a}-\rho_{b}}{\rho_{m a}-\rho_{f}}
$$

\section{Kết luận}

Bài báo giới thiệu quy trình, công thức tính toán được sử dụng cho thí nghiệm chụp cắt lớp 2 mức năng lượng. Những hệ số cần thiết để đưa ra được giá trị $\rho_{\mathrm{b}}$ và $Z_{\text {eff }}$ dựa trên những dữ liệu về số $\mathrm{CT}$ của 2 mức năng lượng cao và thấp.

Việc tính toán được dùng trên 3 mẫu thử và kết quả cho thấy sự khả quan khi sử dụng kỹ thuật này. Việc chụp cắt lớp này cũng được dùng áp dụng cho mẫu lõi đường kính lớn và kết quả được so sánh với giá trị đường cong địa vật lý.

Nghiên cứu này được tiếp tục phát triển để tăng chất lượng của việc chụp cắt lớp 2 mức năng lượng nhằm tính toán các thông số $\rho_{b}$ và $Z_{\text {eff }}$ chính xác hơn. Đồng thời, kỹ thuật chụp cắt lớp cũng cho thấy tiềm năng lớn trong việc xác định các tính chất vật lý của đá chứa cũng như chất lưu vỉa.

Các ứng dụng chụp cắt lớp 2 mức năng lượng nếu được kết hợp với các thông tin từ đường cong địa vật lý và từ biểu đồ phân tích mẫu lõi tiêu chuẩn sẽ cung cấp thông tin hữu ích cho công tác nghiên cứu đặc tính vỉa chứa.

\section{Tài liệu tham khảo}

[1] S.L. Wellington and H.J. Vinegar, "X-ray computerized tomography", Journal of Petroleum Technology, Vol. 39, No. 8, pp. 885 - 898, 1987.

[2] Patricia K. Hunt, Philip Engler, and Caroline Bajsarowicz, "Computed tomography as a core analysis tool: Applications, instrument evaluation, and image improvement techniques", Journal of Petroleum Technology, Vol. 40, No. 9, pp. 1203 - 1210, 1988.

[3] E.M. Withjack, "Computed tomography for rockproperty determination and fluid-flow visualization", SPE Formation Evaluation, Vol. 3, No. 4, pp. 696 - 704, 1988.

[4] A. Kantzas, "Investigation of physical properties of porous rocks and fluid flow phenomena in porous media using computer assisted tomography", In Situ, Vol. 14, No. 1, p. 77 - 132, 1990.

[5] A. Bansal and M.R. Islam, "State-of-the-Art review of nondestructive testing with computer-assisted tomography", International Arctic Technology Conference, Anchorage, AK, 29 - 31 May 1991.

[6] S. Saner, "A review of computer tomography and petrophysical applications (Sabbatical research, 1993 1994)", King Fahd University of Petroleum and Minerals, Dhahran, KSA, 1994.

[7] S. Akin and A.R. Kovscek, "Use of computerized tomography in petroleum engineering research", Annual Report of SUPRI TR 127, Stanford University, Stanford, CA, pp. $63-83,2001$.

[8] E.M. Withjack, C. Devier, and G. Michael, "The role of computed tomography in core analysis", SPE Western Regional/AAPG Pacific Section Joint Meeting, Long beach, CA, 19 - 24 May 2003.

[9] Shameem Siddiqui, James Funk, and Aon Khamees, "Static and dynamic measurements of reservoir heterogeneities in carbonate reservoirs", 2000 SCA Symposium, Abu Dhabi, UAE, 18 - 22 October 2000.

[10] S. Siddiqui, P.J. Hicks, and A.S. Grader, "Verification of buckley-leverett three phase theory using computerized tomography", Journal of Petroleum Science and Engineering, Vol. 15, No. 1, pp. 1 - 21, 1996.

[11] A.Sahni, J.E. Burger, and M.J.Blunt, "Measurement of three- phase relative permeability during gravity drainage using CT scanning", SPE/DOE Improved Oil Recovery Conference, Tulsa, OK, 19 - 22 April 1998.

[12] G.K. Von Schulthness and H-G Smith, Encyclopaedia of edical imaging - Vol. 1: Physics, Technology and Procedures. Martin Dunitz Ltd., London, UK, 1998. 
[13] Q. Lu, "The utility of X-raydual-energytransmission and scatter technologies for Illicit material detection", Ph.D. Dissertation, Virginia Polytechnic Institute, Blacksburg, VA, 1999.

[14] J.S. Gardner and J.L. Dumanoir, "Litho-Density log interpretation", SPWLA Twenty-First Annual Logging Symposium Transactions, 8 - 11 July 1980.

[15] H. Edmundson and L.L. Raymer, "Radioactive logging parameters for common minerals", SPWLA Twentieth Annual Logging Symposium Transactions, 3 - 6 June 1979.
[16] O. Serra, Schlumberger element mineral rock catalog. Schlumberger Publications, 1990.

[17] M. Rider, The geological interpretation of well logs. Gulf Publishing Co., Houston, TX, 1996.

[18] R. Angulo and N. Ortiz, "X-Ray tomography aplications in porous media evaluation", 2nd Latin American Petroleum Engineering Conference (LAPEC), Caracas, Venezuela, 8 - 11 March 1992.

[19] Schlumberger, Log interpretations charts. Schlumberger Publications, 1995.

\section{APPLICATION OF DUAL-ENERGY CT SCAN TO ANALYSE AND PREDICT ROCK PROPERTIES IN CORE ANALYSIS}

\section{Nguyen Lam Quoc Cuong, Nguyen Hong Minh}

Vietnam Petroleum Institute

Email: cuongnlq@vpi.pvn.vn

\section{Summary}

Tomography has been used in the oil and gas industry for more than 20 years to study core sample properties and visualise fluid flow [1 - 8]. Qualitative tomography provides information on heterogeneity [9], petrographic change, fissure, cavity and penetration level of drilling muds, whereas quantitative tomography in addition to the above applications is also used to calculate the bulk density, porosity, and calibration of logging curves.

The article presents the method for prediction of rock properties (such as grain density and porosity, etc.) from the results of CT scans using Dual-energy CT Scanning - DECT at $140 \mathrm{kV}$ and $80 \mathrm{kV}$. The CT scan results are compared with the values of the geophysical curve, showing a great potential in determining the physical properties of the reservoir rock as well as the reservoir fluid.

Key words: Dual-energy CT scanning, rock properties, Compton scattering, photoelectric effect, porosity. 